Article

\title{
Fat Triangulations, Curvature and Quasiconformal Mappings
}

\section{Emil Saucan $^{1,2, *}$ and Meir Katchalski ${ }^{1}$}

${ }^{1}$ Department of Mathematics, Technion, Technion City, Haifa 32000, Israel;

E-Mail: meirk@tx.technion.ac.il

${ }^{2}$ Department of Mathematics and Computer Science, The Open University of Israel, 1 University Rd., Raanana 43107, Israel

* Author to whom correspondence should be addressed; E-Mail: semil@tx.technion.ac.il; Tel.: +972-4-8292896; Fax: +972-4-8294799.

Received: 9 April 2012; in revised form: 31 May 2012 / Accepted: 11 June 2012 /

Published: 4 July 2012

\begin{abstract}
We investigate the interplay between the existence of fat triangulations, $P L$ approximations of Lipschitz-Killing curvatures and the existence of quasiconformal mappings. In particular we prove that if there exists a quasiconformal mapping between two $P L$ or smooth $n$-manifolds, then their Lipschitz-Killing curvatures are bilipschitz equivalent. An extension to the case of almost Riemannian manifolds, of a previous existence result of quasimeromorphic mappings on manifolds due to the first author is also given.
\end{abstract}

Keywords: fat triangulation; Lipschitz-Killing curvatures; quasimeromorphic mapping

\section{Introduction}

The present note is largely motivated by our theorem below, itself a continuation and generalization of previous results of Martio and Srebro [1], Tukia [2] and Peltonen [3]:

Theorem 1.1 ([4]) Let $M^{n}$ be a connected, oriented $n$-dimensional $(n \geq 2)$ submanifold of $\mathbb{R}^{N}$ (for some $N$ sufficiently large), with boundary, having a finite number of compact boundary components, and such that one of the following condition holds:

(i) $M^{n}$ is of class $\mathcal{C}^{r}, 1 \leq r \leq \infty, n \geq 2$;

(ii) $M^{n}$ is a PL manifold and $n \leq 4$;

(iii) $M^{n}$ is a topological manifold and $n \leq 3$. 
Then there exists a non-constant quasimeromorphic mapping $f: M^{n} \rightarrow \widehat{\mathbb{R}^{n}}$, where $\widehat{\mathbb{R}^{n}}=\mathbb{R}^{n} \cup\{\infty\}$ is identified with $\mathbb{S}^{n}$ with spherical metric.

Recall that quasiconformal mappings are defined as follows:

Definition 1.2 Let $(M, d),(N, \rho)$ be metric spaces and let $f:(M, d) \rightarrow(N, \rho)$ be a homeomorphism. Then $f$ is called quasiconformal (or, more precisely, $K$-quasiconformal iff there exists $1 \leq K \leq \infty$, such that, for any $x \in M$, the following holds:

$$
H(f, x)=\limsup _{r \rightarrow 0} \frac{\sup \{\rho(f(x), f(y)) \mid d(x, y)=r\}}{\inf \{\rho(f(x), f(y)) \mid d(x, y)=r\}} \leq K
$$

$H(f, x)$ is called the linear dilatation of $f($ at $x)$.

Obviously, the linear dilatation is a measure of the eccentricity of the image of infinitesimal balls. Therefore (at least if one restricts oneself to Riemannian manifolds) quasiconformal mappings can be characterized as being precisely those maps that

- map infinitesimal balls into infinitesimal ellipsoids (of bounded eccentricity);

- map almost balls into almost ellipsoids;

- distort infinitesimal spheres by a constant factor.

In fact, if one considers (in the Riemannian manifold setting) the linear mapping $f^{\prime}: \mathbb{R}^{n} \rightarrow \mathbb{R}^{n}$, then $f^{\prime}\left(B^{n}\right)=E\left(f^{\prime}\right)$ is an ellipsoid of semi-axes $a_{1} \geq a_{2} \geq \cdots \geq a_{n}$ (and equal to the square roots of the eigenvalue of the adjoint mapping of $f^{\prime}$ ) and the characterizations above follow. Not only this, but, in fact,

$$
H(A)=\frac{a_{1}}{a_{n}}
$$

Moreover, quasiconformal mappings

- distort local distances by a fixed amount;

- preserve approximative shape.

(However, in these cases the characterization is not sharp, the proper class of functions characterized by this property being the so-called quasisymmetric mappings.)

Of course, one naturally asks whether the "quasiconformal" in Definition 1.2 above implies, indeed, as the name suggests, that quasiconformal mappings "almost" preserve angles (given that conformal mappings do). The answer is, as expected, positive—-see [5].

Remark 1.3 There exist two other definitions of quasiconformality (for mappings between Riemannian manifolds of the same dimensionality), but we have chosen the one above-the so called metric definition-due to its simplicity, naturalness in our context (see below) and the fact that it makes sense for any metric space. On the other hand, if one wishes to prove even the simplest, intuitive geometric properties (like the ones mentioned above), a delicate interplay of all of the three definitions is needed.

Generalizing the definition of quasiconformality to maps that fail to be homeomorphisms necessitates a number of additional preliminary definitions and notations: 
Definition 1.4 Let $(M, d),(N, \rho)$ be metric spaces. $f:(M, d) \rightarrow(N, \rho)$ is called

(i) open iff it maps open sets onto open sets;

(ii) discrete iff $f^{-1}(y)$ is discrete (in $M$ ), for any $y \in N$.

Definition 1.5 The $M, N$ and $f$ be as above. The branch set $B_{f}$ of $f$ (also called the critical set in some of the literature) is defined as

$$
B_{f}=\{x \in M \mid f \text { is not locally homeomorphic at } x\}
$$

To introduce a fitting metric definition of quasiregularity, we have to restrict somewhat the class of spaces on which they are defined. However, this class still represents a generalization of more established definitions and more than suffices for our geometric purposes here.

Definition 1.6 Let $(M, d),(N, \rho)$ be metric spaces. $f:(M, d) \rightarrow(N, \rho)$ is called

(i) quasiregular (or, more precisely, K-quasiregular) iff

1. It is sense preserving, open and discrete;

2. $H(f, x)$ is locally bounded;

3. There exists $H_{0}<\infty$ such that $H(f, x) \leq H_{0}$ for a.e. $x \in B_{f}$.

(iii) quasimeromorphic iff $(N, \rho)$ is the unit sphere $\mathbb{S}^{n}$ (equipped with standard metric).

Note that, by [6], Theorem 6.2, the definition above, coincides in the classical case with the other, more common definitions of quasiregularity. We have preferred here to introduce this, rather than other, more common and established definitions, because, in fact, we mainly need the metric distortion properties, thence in our context its simplicity has great appeal. Moreover, it highlights the fact that quasiregular mappings are, in fact, quite general mappings that satisfy only a set of rather natural topological and metric conditions.

Since the theorem above concerns the existence of quasiconformal mappings, which, as we have seen, are generalizations of quasiconformal mappings and since Proposition 3.3 below concerns the existence of quasiconformal mappings between almost Riemannian manifolds (see Definition 3.1 below), their presence in the title is elucidated.

Given that the proof of Theorem 1.1 essentially requires the construction of a "chess-board" fat triangulation (followed by the alternate quasiconformal mapping of the "black" and "white" simplices to the interior, respective exterior of the standard simplex in $\mathbb{R}^{n}$ ), the presence in the title of the "fat triangulations" is also explained. Moreover, it follows that to prove Theorem 1.1 one has first to ensure the existence of fat triangulations on manifolds. The required result is given below:

Theorem 1.7 ([4]) Let $M^{n}$ be a Riemannian manifold satisfying the conditions in the statement of Theorem 1.1 above.

If the boundary components admit fat triangulations of fatness $\geq \varphi_{0}$, then there exists a global fat triangulation of $M^{n}$. 
Remark 1.8 In fact, the conditions on the compactness and boundedness of the boundary components in the theorem above are too strong, as indicated by the results in [7,8], where the theorem above was shown to hold also for (hyperbolic) manifolds with infinitely many boundary components (as well as for more general spaces). The role of the conditions in question is to exclude certain "pathological" cases.

Given the triangulation results of $[9,10]$ for manifolds without boundary, the following corollary follows immediately:

Corollary 1.9 ([4]) Let $M^{n}$ be a Riemannian manifold satisfying the conditions in the statement of Theorem 1.1 above.

Then $M^{n}$ admits a fat triangulation.

Remark 1.10 For a similar result see [11].

Recall that fat triangulations (also called thick in some of the literature) are defined (in [10]) as follows:

Definition 1.11 Let $\tau \subset \mathbb{R}^{n} ; 0 \leq k \leq n$ be a $k$-dimensional simplex. The fatness $\varphi$ of $\tau$ is defined as being:

$$
\varphi=\varphi(\tau)=\inf _{\substack{\sigma<\tau \\ \operatorname{dim} \sigma=j}} \frac{\operatorname{Vol}_{j}(\sigma)}{\operatorname{diam}^{j} \sigma}
$$

The infimum is taken over all the faces of $\tau, \sigma<\tau$, and $\operatorname{Vol}_{j}(\sigma)$ and $\operatorname{diam} \sigma$ stand for the Euclidean $j$-volume and the diameter of $\sigma$ respectively. (If $\operatorname{dim} \sigma=0$, then $\operatorname{Vol}_{j}(\sigma)=1$, by convention.) A simplex $\tau$ is $\varphi_{0}$-fat, for some $\varphi_{0}>0$, if $\varphi(\tau) \geq \varphi_{0}$. A triangulation (of a submanifold of $\mathbb{R}^{n}$ ) $\mathcal{T}=\left\{\sigma_{i}\right\}_{i \in \mathbf{I}}$ is $\varphi_{0}$-fat if all its simplices are $\varphi_{0}$-fat. A triangulation $\mathcal{T}=\left\{\sigma_{i}\right\}_{i \in \mathbf{I}}$ is fat if there exists $\varphi_{0} \geq 0$ such that all its simplices are $\varphi_{0}$-fat.

The following result gives a more intuitive interpretation on the notion of fatness of simplices as a function of their dihedral angles in all dimensions:

Proposition 1.12 ([10]) There exists a constant $c(k)$ that depends solely upon the dimension $k$ of $\tau$ such that

$$
\frac{1}{c(k)} \cdot \varphi(\tau) \leq \min _{\sigma<\tau} \measuredangle(\tau, \sigma) \leq c(k) \cdot \varphi(\tau)
$$

and

$$
\varphi(\tau) \leq \frac{\operatorname{Vol}_{j}(\sigma)}{\operatorname{diam}^{j} \sigma} \leq c(k) \cdot \varphi(\tau)
$$

Having explained our concern with fat triangulation and quasimeromorphic mappings, we still have to explain the connection with differential geometry. The inherent relation between the existence of fat triangulations and differential geometry is expressed by the essential role of curvature in the construction of such triangulations. This ingrained connection is transparent in the very basic proof of Peltonen [3] that represents one of the basic ingredients of our own construction [4]. More precisely, an interplay between intrinsic curvature (preserved during the Nash embedding process employed) and extrinsic curvature (via the tubular radius, see [3]) is used to obtain the desired fat triangulation. We have investigated in more detail this aspect of the role of curvature, and showed the possibility of constructing 
fat triangulations using solely intrinsic curvature, Ricci curvature, to be more precise, in $[12,13]$ and, in a more general context, in [14].

The reverse direction, that is the role of fat triangulations in determining (in the $P L$ case) or approximating (in the smooth case) curvature(s) was shown in detail in [10]—-see Theorem [10] below. It is this direction, and its connection with the existence of quasimeromorphic mappings, that we explore in this paper.

Theorem 1.13 ([10]) Let $M^{n}$ be a compact Riemannian manifold, with or without boundary, and let $M_{i}^{n}$ be a sequence of fat PL (piecewise flat) manifolds, that are secant approximations of $M^{n}$, converging to $M^{n}$ in the Hausdorff metric. Denote by $\mathfrak{R}$ and $\mathfrak{R}_{i}$ respectively, the Lipschitz-Killing curvatures of $M^{n}$, $M_{i}^{n}$. Then $\mathfrak{R}_{i} \rightarrow \mathfrak{R}$ in the sense of measures.

Recall that the Hausdorff metric is defined as follows:

Definition 1.14 Let $(X, d)$ be a metric space and let $A, B \subseteq X$. The Hausdorff distance between $A$ and $B$ is defined as:

$$
d_{H}(A, B)=\inf \left\{r>0 \mid A \subset U_{r}(B), B \subset U_{r}(A)\right\}
$$

where $U_{r}(A)$ denotes the r-neighborhood of $A$, i.e., $U_{r}(A)=\bigcup_{a \in A} B(a, r)$. (Here, $B(a, r)$, denotes, as usually, the open ball of center a and radius $r$.)

(Note that, since $M^{n}$ is compact and since, given that the $P L$ manifolds $M_{i}^{n}$ are secant approximations of $M^{n}$, all these manifolds can be considered to be embedded in the same $\mathbb{R}^{N}$. Thus we can employ the Hausdorff metric, instead of the more abstract Gromov-Hausdorff metric.)

The convergence of measure considered here is the weak convergence:

Definition 1.15 Let $X$ be a complete, separable metric space, equipped with its Borel $\sigma$-algebra, and let $\left\{\mu_{i}\right\}_{i}$ be of sequence of Borel measures of finite mass on $X$. The sequence $\left\{\mu_{i}\right\}_{i}$ is said to converge (weakly) to a measure $\mu$ iff $\int_{X} f d \mu_{i} \rightarrow \int_{X} f d \mu$, for any bounded, positive and continuous function $f: X \rightarrow \mathbb{R}$.

Recall also that, for a Riemannian manifold $M^{n}$, the Lipschitz-Killing curvatures are defined as follows:

$$
\begin{gathered}
R^{j}\left(M^{n}\right)=\frac{1}{(n-j) ! 2^{j} \pi^{j / 2}(j / 2) !} \sum_{\pi \in S_{n}}(-1)^{\epsilon(\pi)} \Omega_{\pi(1) \pi(2)} \wedge \cdots \wedge \Omega_{\pi(j-1) \pi(j)} \wedge \omega_{\pi(j+1)} \wedge \\
\wedge \cdots \wedge \omega_{\pi(n)}
\end{gathered}
$$

where $\Omega_{\pi(j-1) \pi(j)}$ are the curvature 2-forms and $\omega_{k l}$ denote the connection 1-forms, and they are interrelated by the structure equations:

$$
\left\{\begin{array}{l}
d \omega_{k}=-\sum_{i} \omega_{k l} \wedge \omega_{l} \\
d \omega_{k l}=-\sum_{i} \omega_{k i} \wedge \omega_{i l}+\Omega_{k l}
\end{array}\right.
$$

where $\left\{\omega_{k}\right\}$ is the dual basis of $\left\{e_{k}\right\}$.

Remark 1.16 The low dimensional Lipschitz-Killing curvatures are, in fact, quite familiar: $R^{0} \equiv$ volume and $R^{2} \equiv$ scalar curvature. Moreover, $R^{n} \equiv$ Gauss-Bonnet-Chern form, $($ for $n=2 k)$. 
Remark 1.17 The integral $\int_{M^{n}} R^{j}$ is also known as the integrated mean curvature (of order $j$ ).

In a similar manner (but technically slightly more complicated), one can define the associated boundary curvatures (or mean curvatures) $H^{j}$ which are curvature measures on $\partial M^{n}$ : Let $\left\{e_{k}\right\}_{1 \leq k \leq n}$ be an orthonormal frame for the tangent bundle $T_{M^{n}}$ of $M^{n}$, such that, along the boundary $\partial M^{n}, e_{n}$ coincides with the inward normal. Then, for any $2 k+1 \leq j \leq n$, we define

$$
H^{j}=\sum_{k} \Omega_{j, k}
$$

where

$$
\begin{aligned}
\Omega_{j, k}=c_{j, k} \sum_{\pi \in S_{n-1}}(-1)^{\epsilon(\pi)} \Omega_{\pi(1) \pi(2)} \wedge \cdots & \wedge \Omega_{\pi(2 k-1) \pi(2 k)} \wedge \omega_{\pi(2 k+1), n} \wedge \cdots \wedge \omega_{\pi(j-1), n}, \omega_{\pi(j)} \wedge \\
& \wedge \cdots \wedge \omega_{\pi(n-1)}
\end{aligned}
$$

and

$$
c_{j, k}= \begin{cases}(-1)^{k}\left(2^{j} \pi^{\frac{j-1}{2}} k !\left(\frac{j-1}{2}-k\right) !(n-j) !\right)^{-1}, & j=2 p+1 \\ (-1)^{k}\left(2^{k+\frac{j}{2}} \pi^{\frac{j}{2}} k !(j-2 k-1) !(n-j) !\right), & j=2 p\end{cases}
$$

These curvatures measures are normalized by imposing the condition that:

$$
\int_{T^{n-j} \times M^{j}} R^{j}+\int_{T^{n-j} \times \partial M^{j}} H^{j}=\chi\left(M^{j}\right) \operatorname{Vol} T^{n-j}
$$

for any flat $T^{n-j}$.

Remark 1.18 As is the case with the Lipschitz-Killing curvatures, the low dimensional boundary curvatures also have quite familiar interpretations: $H^{1} \equiv$ area boundary, $H^{2} \equiv$ mean curvature for inward normal (as expected given the generic names for these $H^{j}-s$ ), etc.

Remark 1.19 One can fatly triangulate the smooth manifold $M^{n}$ and obtain the desired approximation results for curvatures using the intrinsic metric, not just PL (Euclidean) approximations (see [10] and, for a generalization, [15]).

The need for fat triangulations as a prerequisite for Theorem 1.13 should not be surprising, in view of the characterization of fat triangulations as being those triangulations having dihedral angles bounded from below (Proposition 1.12) and in view of the following expression of the Lipschitz-Killing curvatures in terms of dihedral angles (see [10] for the proof):

$$
\begin{gathered}
R^{j}=\sum_{\sigma^{n-j}}\left\{1-\chi\left(L\left(\sigma^{j}\right)\right)+\sum_{l} \measuredangle\left(\sigma^{n-j}, \sigma^{n-j+i_{1}}\right) \cdots \measuredangle\left(\sigma^{n-j+i_{1-1}}, \sigma^{n-j+i_{1}}\right)\right. \\
\left.\cdot\left[1-\chi\left(L\left(\sigma^{n-j+i_{l}}\right)\right)\right]\right\} \operatorname{Vol}\left(\sigma^{n-j}\right)
\end{gathered}
$$

where $L\left(\sigma^{j}\right)$ denotes the (spherical) link of $\sigma^{j}$, and $\measuredangle\left(\sigma^{i}, \sigma^{j}\right)$ is the internal dihedral angle of $\sigma^{i}<\sigma^{j}$; $\measuredangle\left(\sigma^{i}, \sigma^{j}\right)=\operatorname{Vol}\left(L\left(\sigma^{i}, \sigma^{j}\right)\right.$, where the volume is normalized such that $\operatorname{Vol}\left(\mathbb{S}^{n}\right)=1$, for any $n$. (See [10] for further details.) (Here $\chi$, Vol denote, as usual, the Euler characteristic and volume of $\sigma^{k}$, respectively.)

The differential geometric consequence of Theorems 1.13 and 1.7, as well as Corollary 1.9 is the following: 
Theorem 1.20 ([15]) Let $N=N^{n-1}$ be a not necessarily connected manifold, such that $N=\partial M$, $M=M^{n}$, where $M^{n}$ is, topologically, as in the statement of Theorem 1.7.

(i) If $M, N$ are PL manifolds, then the Lipschitz-Killing curvature measures of $N$ can be extended to those of $M$. More precisely, there exist Lipschitz-Killing curvature measures $\Re=\left\{R^{j}\right\}$ on $\bar{M}=M \cup N$, such that $\left.\mathfrak{R}\right|_{N}=\mathfrak{R}_{N}$ and $\left.\mathfrak{R}\right|_{M}=\mathfrak{R}_{M}$, except on a regular (arbitrarily small) neighbourhood of $N$, where $\mathfrak{R}_{N}, \mathfrak{R}_{M}$ denote the curvature measures of $N, M$ respectively.

(ii) If $M, N$ are smooth manifolds, then the same holds, but only in the sense of measures.

Remark 1.21 Recall that $\left.R^{j}\right|_{\partial M^{n}}=H^{j}$ and, in the case of PL manifolds, it represents the contribution of the $(n-j)$-dimensional simplices that belong to the boundary. (For an explicit formula, see any of the formulas (3.23), (3.38) or (3.39) of [10].)

Remark 1.22 In a sense, the theorem above can be considered, in view of the previous Remark, as the "reverse" of the result of [10], Section 8, regarding the convergence of the boundary measures.

\section{Quasiconformal Mappings Between Manifolds}

Our main result is the observation that spaces that admit "good" curvature convergence in secant approximation are geometric branched covers of $\mathbb{S}^{n}$. More precisely, from the considerations above we obtain immediately the following theorem:

Theorem 2.1 Let $M_{1}^{n}, M_{2}^{n}$ be two (connected) manifolds, topologically as in the statement of Theorem 1.1, and let $\varphi: M_{1}^{n} \rightarrow M_{2}^{n}$ be a K-quasiconformal mapping.

(i) If $M_{1}^{n}, M_{2}^{n}$ are PL manifolds, then there exists $C(K, n, j)>0$, such that

$$
\frac{1}{C(K, n, j)}\left|R_{1}^{j}\right| \leq\left|R_{2}^{j}\right| \leq C(K, n, j)\left|R_{1}^{j}\right|
$$

where $R_{1}^{j}, R_{2}^{j}$ denote the Lipschitz-Killing curvatures of $M_{1}^{n}, M_{2}^{n}$, respectively.

(ii) If $M_{1}^{n}, M_{2}^{n}$ are smooth manifolds, then the same double inequality holds, but only in the sense of measures.

Proof (i) We should first underline the fact that, in the $P L$ case, the quasiconformal mappings under study are, by definition, $P L$ homeomorphisms, thence, again by definition, they are linear on some subdivision of $M_{1}^{n}$ (see [9]). Consequently, they do not preserve, in general, combinatorial structure. From this it follows, in particular, that a point into the interior of an $n$-simplex of $M_{1}^{n}$ can be mapped to a vertex of $M_{2}^{n}$. (One such example—in a sense generic (see [9])—would be the barycenter of (a face of) some $\sigma_{1}^{n} \in M_{n}^{1}$ and its mapping after such a subdivision.)

Now, from Agard's characterization of quasiconformal mappings [5] and from Proposition 12 it follows that angles are distorted by a factor of $K$, i.e.,

$$
\frac{1}{K} m(\alpha) \leq m(f(\alpha)) \leq K m(\alpha)
$$

where $m(\alpha)$ denotes the measure of the angle $\alpha$. This follows immediately from [16], Theorem 4.1 and from the fact that the inverse of a $K$-quasiconformal map is also $K$-quasiconformal. Since the dihedral 
angles in any dimension $j \leq n$ can be expressed, hierarchically, as a function of the (planar) angles of its faces, we obtain that there exists $C=C(K, j)$, such that

$$
\frac{1}{C(K, j)} m_{j-1}\left(\alpha_{j}\right) \leq m_{j}\left(f\left(\alpha_{j}\right)\right) \leq C(K, j) m_{j-1}\left(\alpha_{j}\right)
$$

where $m_{j-1}$ denotes the $(j-1)$-dimensional measure (content) of the ( $j$-dimensional dihedral) angle $\alpha_{j}$. (Alternatively, by making appeal to [5], Section 6, one can obtain the double inequality above directly in terms of $K$ itself, albeit at the precise of loosing intuitiveness.) But, by Formula (12), the Lipschitz-Killing curvatures $R^{j}$ of a $P L$ (piecewise flat) manifold are functions of the measures of the dihedral angles in dimension $\geq n-j$ (and, implicitly, on the dimension $n$ of the manifold), thence there exists $C_{1}=C_{1}(K, n, j)$ such that Formula (13) holds.

That Formula (13) holds, in general, only for the absolute values of the curvatures $R^{j}$, is a consequence of the fact, already noted above, that interior points - that is of 0 curvature-can be mapped into (essential) vertices-i.e., carriers of positive or negative curvature. Note that the respective inequalities for the $R^{j}$ 's- that is without passing to the absolute values - can be obtained if these curvatures are bounded away from 0 and the dilatation $K \approx 1$, where "closeness to 1 " is a function of lower/upper bounds on the curvatures $R^{j}$.

(ii) By our triangulation result above, namely Corollary 1.6, there exists a fat triangulation of $M^{n}$. Moreover, by repeated (almost) parallel (or median) subdivisions (i.e., obtained dividing any given simplices into $k^{2}$ smaller ones, by hyper-planes (lines, for $n=2$ ) parallel to the faces of given the simplex), the mesh of the triangulation can be made arbitrarily small, while ensuring that the fatness of the elements of a such sequence of subdivisions $\left\{M_{i}^{n}\right\}$ is uniformly bounded from below, i.e., there exists $\varphi_{0}>0$, such that $\varphi_{i}=\varphi\left(M_{i}^{n}\right) \geq \varphi_{0}$, for all $i \in \mathbb{N}$. (Note that in fact, the construction requires such repeated subdivisions—see [4].) This, in conjunction with [10], Theorem 5.1 (for manifolds without boundary and the interior of manifolds with boundary) and Theorem 8.1 (for the case of the boundary curvatures) assures the existence of arbitrarily good approximations in measure of the Lipschitz-Killing curvatures $R^{j}$ of the smooth manifold $M^{n}$ by those of a sequence of $P L$ approximations $\left\{M_{i}^{n}\right\}$. The desired result now follows immediately from (1).

Remark 2.2 As noted above, fatness of the elements of the approximating sequence $\left\{M_{i}^{n}\right\}$ is an essential ingredient in assuring the convergence of the curvature measures to that of the smooth manifold $M^{n}$. We should emphasize that this is, however, only an approximation in measure and the error $\left|R^{j}(U)-R_{i}^{j}(U)\right|$ is a function not only on the curvature tensor of $M^{n}$ and of its gradient, but also of $\varphi_{0}$ and on the mesh of the triangulation, and, of course, of the volume of the neighbourhood U (of a given vertex) where curvatures are approximated-see [10] Formula (5.4). However, in many applications (see, e.g. [17]), one wishes to estimate the error in the approximation of $R^{j}$ at a given vertex. For this one has to take into account the error, as a function of the mesh of the simplices incident to a vertex $v$ of the sides and angles of these simplices-see [10], Formula (1.21) and Formula (4.7) and Appendix A2, respectively. Moreover, the change of dihedral angles of these approximations is (as expected) a function of the sectional curvatures at v-see [10], Formula (5.10).

Alternative Proof In this approach, one regards the relevant edges of a fine enough triangulation both as 
the principal vectors and as semi-axes of an "infinitesimal" ellipsoid and apply directly the definition of quasiconformality, or rather its geometric interpretations (see Section 1 above, in particular Formula 2), in conjunction with the following expression of the Lipschitz-Killing curvatures:

$$
R^{j}\left(M^{n}\right)=\frac{1}{\operatorname{Area}\left(\mathbb{S}^{n-j-1}\right)} \int_{M^{n-1}} S_{n-j-1}\left(k_{1}(x), k_{2}(x), \ldots, k_{n-1}(x)\right) d \mathcal{H}^{n-1}
$$

where $M^{n-1}=\partial M^{n}, d \mathcal{H}^{n-1}$ denotes the $(n-1)$-dimensional Hausdorff measure, and where the symmetric functions $S_{j}$ are defined by:

$$
S_{j}\left(k_{1}(x), k_{2}(x), \ldots, k_{j-1}(x)\right)=\sum_{1 \leq k_{i_{1}} \leq k_{i_{k}} \leq j-1} k_{i_{1}}(x) \cdots k_{i_{k}}(x)
$$

$k_{1}(x), k_{2}(x), \ldots, k_{n-1}(x)$ being the principal curvatures-see e.g. [18].

(i) For $P L$ manifolds, this approach would be somewhat naive, if applied directly, as it gives only approximative results. (This is a consequence of the fine interplay between the necessity of ensuring the fatness of the triangulation, simultaneously with a good sampling of the direction in the tangential plane (or rather cone)—see [15].) One can overcome this obstacle by using an extension of the notion of principal curvatures to a far larger class of geometric objects than mere smooth or even $P L$ manifolds (see, e.g. [18]), by passing to the so-called generalized principal curvatures:

First, let us note that, for small enough $\varepsilon>0, \partial X_{r}$ is a $\mathcal{C}^{1,1}$-hypersurface. Therefore, they admit principal curvatures (in the classical sense) $k_{i}^{\varepsilon}(x+\mathbf{n})$ at almost any point $p=x+\mathbf{n}$, where $\mathbf{n}$ denotes the normal unit vector (at $x)$. Define the generalized principal curvatures by: $k_{i}(\varepsilon, \mathbf{n})=k_{i}^{\varepsilon}(x+\mathbf{n})$. Then $k_{i}(\varepsilon, \mathbf{n})$ exist $\mathcal{H}^{n-1}$-a.a. $(x, \mathbf{n})$.

Note that, in particular, $\varepsilon$ has to be strictly smaller than the reach of $X$,

$$
\operatorname{reach}(X)=\sup \left\{r>0 \mid \forall y \in X_{r}, \exists ! x \in X \text { nearest to } y\right\}
$$

where $X_{r}$ denotes the $r$-neighbourhood of $X$, and that the reach itself has to be strictly positive.

Using this generalization of principal curvatures, one can retrieve a proper analogue of Formula 14, namely

$$
C_{k}(X, B)=\int_{\operatorname{nor}(X)} \mathbf{1}_{B} \prod_{i=1}^{d-1} \frac{1}{\sqrt{1+\kappa_{i}(x, \mathbf{n})^{2}}} S_{d-1-k}\left(\kappa_{1}(x, \mathbf{n}), \cdots, \kappa_{d-1}(x, \mathbf{n})\right) d \mathcal{H}^{d-1}(x, \mathbf{n})
$$

where $C_{k}(X, B)$ denote the so called Lipschitz-Killing curvature measures (see [18] and the bibliography therein for details), and nor $(X)$ denotes the (unit) normal bundle of $X$ :

$$
\operatorname{nor}(X)=\left\{(x, \mathbf{n}) \in \partial X \times \mathbb{S}^{d-1} \mid \mathbf{n} \in \operatorname{Nor}(X, x)\right\}
$$

where $\left.\operatorname{Nor}(X, x)=\left\{\mathbf{n} \in S_{d-1} \mid<\mathbf{n}, v>\leq 0, v \in \operatorname{Tan}(X, x)\right)\right\}$ is the normal cone (to $X$ at the point $x \in T$ ), dual to the tangent cone (to $X$ at the point $x \in T$ ).

Using the convergence properties of the generalized principal curvatures and of the Lipschitz-Killing curvature measures (again, see [18] and the bibliography therein), the result now follows along the lines of the second part of our first proof of the theorem. 
(ii) The case of smooth manifolds follows immediately following the same scheme as in the previous proof.

Remark 2.3 Formula (14) above shows why the Lipschitz-Killing curvatures are also called the total mean curvatures and the " $S_{j}$ "-s are called the mean curvatures (of order $j$ ).

\section{Quasimeromorphic Mappings on Almost Riemmanian Manifolds}

We also bring here a generalization of Theorem 1.1, but, before stating this next result, we need to introduce (following [19]) yet another definition

Definition 3.1 A metric space $(M, d)$ is called an almost Riemannian space iff

1. $M$ is a smooth manifold;

2. There exists a (smooth) Riemannian metric $g$ on $M$ and a constant $C_{0}>0$, such that, for any $x \in M$, there exists a neighbourhood $U(x)$, such that

$$
C_{0}^{-1} d(y, z) \leq \operatorname{dist}_{g}(y, z) \leq C_{0} d(y, z)
$$

for all $y, z \in U(x)$.

The basic - and motivational-example of an almost metric space (beyond the trivial one $d \equiv g$ ) is given by any smooth (Riemannian) manifold embedded in some $\mathbb{R}^{N}$, with $d$ being the Euclidean distance in $\mathbb{R}^{N}, d=$ dist $_{E u c l}$, i.e., precisely the setting which we were concerned in the previous section: The secant approximation of an embedded smooth manifold, with its Euclidean (ambient) metric is an almost Riemannian manifold (relative, so to say, to the approximated smooth manifold).

The relevant result here is the following theorem that was proved in [15]:

Theorem 3.2 ([15]) Let $(M, d)$ be an almost Riemannian manifold, where $M$ satisfies the conditions in the statement of Theorem 1.1. Then it admits a fat triangulation.

The theorem above and the construction technique of quasimeromorphic mappings employed in [4] and briefly described in Section 1 above, immediately imply the following:

Proposition 3.3 Let $(M, d)$ be a connected almost Riemannian manifold, topologically as in the statement of Theorem 1.1. Then there exists a non-constant quasimeromorphic mapping $f: M \rightarrow \widehat{\mathbb{R}^{n}}$.

Remark 3.4 For other generalizations of Theorems 1.7 and 1.1, see [8,14]. 


\section{Final Remarks}

We do not consider here the problem of extending Proposition 2.1 to the case of general quasiregular mappings, as well as discussing other related aspects relating quasiregular mappings and curvature, but we rather postpone them for further study (see [20]).

We conclude, therefore, this paper with the following Remark and the ensuing Question:

Remark 4.1 Since all the theorems for geometric branched coverings of $\mathbb{S}^{n}$ were obtained via the Alexander Trick, i.e., by constructing fat chessboard triangulation one is easily conducted to the following:

Question 1 Does $M^{n}$ admit a qr-mapping on $\mathbb{S}^{n}$ iff it admits "good" curvature convergence in secant approximation?

\section{Acknowledgements}

First author's research supported by European Research Council under the European Community's Seventh Framework Programme (FP7/2007-2013) / ERC grant agreement $n^{\circ}$ [203134] and by ISF grants 221/07 and 93/11.

The authors would like to thank the referees for their many helpful corrections, comments and suggestions.

\section{References}

1. Martio, O.; Srebro, U. On the existence of automorphic quasimeromorphic mappings in $R^{n}$. Ann. Acad. Sci. Fenn. Ser. I Math. 1977, 3, 123-130.

2. Tukia, P. Automorphic quasimeromorphic mappings for torsionless hyperbolic groups. Ann. Acad. Sci. Fenn. Math. 1985, 10, 545-560.

3. Peltonen, K. On the existence of quasiregular mappings. Ann. Acad. Sci. Fenn. Ser. I Math. Diss. 1992, 85, 1-48.

4. Saucan, E. Note on a theorem of Munkres. Mediterr. J. Math. 2005, 2, 215-229.

5. Agard, S.B. Angles and quasiconformal mappings in space. J. Anal. Math. 1969, 22, 177-200.

6. Rickman, S. Quasiregular Mappings; Springer-Verlag: Berlin/Heidelberg, Germany, 1993.

7. Saucan, E. The existence of quasimeromorphic mappings. Ann. Acad. Sci. Fenn. Math. 2006, 31, 131-142.

8. Saucan, E. Remarks on the existence of quasimeromorphic mappings. Cont. Math. 2008, 455, 325-331.

9. Munkres, J.R. Elementary Differential Topology; Princeton University Press: Princeton, NJ, USA, 1966.

10. Cheeger, J.; Müller, W.; Schrader, R. On the curvature of piecewise flat spaces. Comm. Math. Phys. 1984, 92, 405-454.

11. Cairns, S.S. On the triangulation of regular loci. Ann. Math. 1934, 35, 579-587.

12. Saucan, E. Intrinsic differential geometry and the existence of quasimeromorphic mappings. Revue Roumaine de Math. Pures et Appl. 2009, 54, 565-574. 
13. Saucan, E.; Katchalski, M. The existence of thick triangulations-an "elementary" proof. Open Math. J. 2009, 2, 8-11.

14. Saucan, E. Curvature based triangulation of metric measure spaces. Cont. Math. 2011, 554, 207-227.

15. Saucan, E. Fat triangulations and differential geometry. arXiv:1108.3529v1 [math.DG]. Available online: http://arxiv.org/abs/1108.3529 (accessed on 13 June 2012).

16. Agard, S.B.; Gehring, F.W. Angles and quasiconformal mappings. Proc. Lond. Math. Soc. 1965, $14 A, 1-21$.

17. Saucan, E.; Appleboim, E.; Zeevi, Y.Y. Image projection and representation on $S^{n}$. J. Fourier Anal. Appl. 2007, 13, 711-727.

18. Zähle, M. Curvature theory for singular sets in euclidean spaces. Unpublished work, 2005.

19. Semmes, S. Finding curves on general spaces through quantitative topology, with applications to Sobolev and Poincaré inequalities. Sel. Math. 1996, 2, 155-295.

20. Saucan, E. Fat Triangulations and Quasiregular Mappings. Unpublished work, 2012.

(c) 2012 by the authors; licensee MDPI, Basel, Switzerland. This article is an open access article distributed under the terms and conditions of the Creative Commons Attribution license (http://creativecommons.org/licenses/by/3.0/). 